\title{
Unravelling Irradiation-Induced Detwinning Mechanisms via In Situ and Aberration-Corrected TEM combined with Atomistic Simulations
}

\author{
Pranav K. Suri ${ }^{1}$, James E. Nathaniel, $\mathrm{II}^{1}$, Elaf A. Anber ${ }^{1}$, Meimei Li $^{2}$, Marquis A. Kirk ${ }^{2}$, Jianguo \\ Wen $^{3}$, and Mitra L. Taheri ${ }^{1}$ \\ 1. Department of Materials Science and Engineering, Drexel University, Philadelphia, PA, USA \\ 2. Nuclear Engineering Division, Argonne National Laboratory, Lemont, IL, USA \\ 3. Center for Nanoscale Materials, Argonne National Laboratory, Lemont, IL, USA
}

Next generation nuclear energy systems require advanced structural materials having superior radiation tolerance to increase their longevity and robustness while maintaining optimum performance. In this quest, various materials design of interfaces has been studied to understand efficiency of radiation-induced defect absorption [1]. Nanostructured materials are of particular interest due to an extremely high density of interfaces [1]. Nanotwins are bands of multiple twin boundaries spaced nanometers apart; twin boundaries are a special set of interfaces that can exhibit properties atypical of normal grain boundaries. For instance, a nanotwinned structure in diamonds exhibits enhanced hardness and thermal stability compared to a pure diamond structure [2]. Moreover, nanotwinned metals have shown intriguing radiation response making twin boundaries an attractive tuning parameter for advanced structural materials [3], however, the structural stability of nanotwins with respect to radiation damage is not completely understood.

Under ion irradiation, nanotwinned metals have displayed detwinning due to the absorption of radiation-induced defects. Detwinning results in the retraction of incoherent twin boundaries (ITB) and the decimation of coherent twin boundaries (CTB) [4]; proposed models and experimental results attempt to explain this behavior [4,5], however, atomic-resolution investigations of detwinning mechanisms lack experimental substantiation due to resolution limits of in situ TEM ion irradiation experiments. Here, we describe our efforts to investigate ion irradiation assisted detwinning mechanisms in nanotwinned metals at unprecedented spatial and temporal resolutions via spherical and chromatic aberration-corrected TEM imaging combined with in situ TEM ion irradiation videos. The experimental results are coupled with atomistic simulations to explore possible detwinning mechanisms. We perform in situ TEM ion irradiation in copper bicrystals containing sparsely distributed nanotwins with $1 \mathrm{MeV} \mathrm{Kr}^{2+}$ at IVEM-Tandem facility, Argonne National Laboratory. Atomic-resolution imaging is conducted in FEI Titan 80300 ST equipped with CEOS $\mathrm{C}_{\mathrm{c}} / \mathrm{C}_{\mathrm{s}}$ corrector on the imaging side of the column.

Detwinning involves an abrupt motion of ITB and occurs in a slip-stick fashion by absorbing defects clusters [4,5]. For example, Figure 1 (left panel) shows the snapshots of a nanotwin undergoing detwinning with continuous ion irradiation but the time-interval of ITB retraction is non-linear. Under continuous ion beam irradiation, detwinning ceases even though there is a significant increase in the defect density around the detwinned structure (Figure 1(right panel)). In addition, we find a $14 \mathrm{~nm}$ wide ITB in the detwinned structure (Figure 2) which is resistant to further detwinning. The implications for these and other results explaining the structural stability of nanotwins towards ion irradiation will be discussed in the context of atomistic mechanisms for slip-stick behavior observed in detwinning and determination of the conditions for initiation and decimation of detwinning [6]. 
References:

[1] I. J. Beyerlein et al., Prog. Mater. Sci. 74 (2015), 125.

[2] Q. Huang et al., Nature 510 (2014), 250.

[3] K. Y. Yu et al., Nat. Commun. 4 (2013), 1377.

[4] K. Y. Yu et al., Scr. Mater. 69 (2013), 385.

[5] J. Du et al., Sci. Technol. Adv. Mater. (2018), just accepted.

[6] This work was supported by the U.S. Department of Energy, Office of Nuclear Energy under DOE Idaho Operations Office Contract DE-AC07- 051D14517 as part of a Nuclear Science User Facilities experiment. We acknowledge Kenneth McClellan and Darrin Byler from Los Alamos National Laboratory for providing us with copper bicrystals.
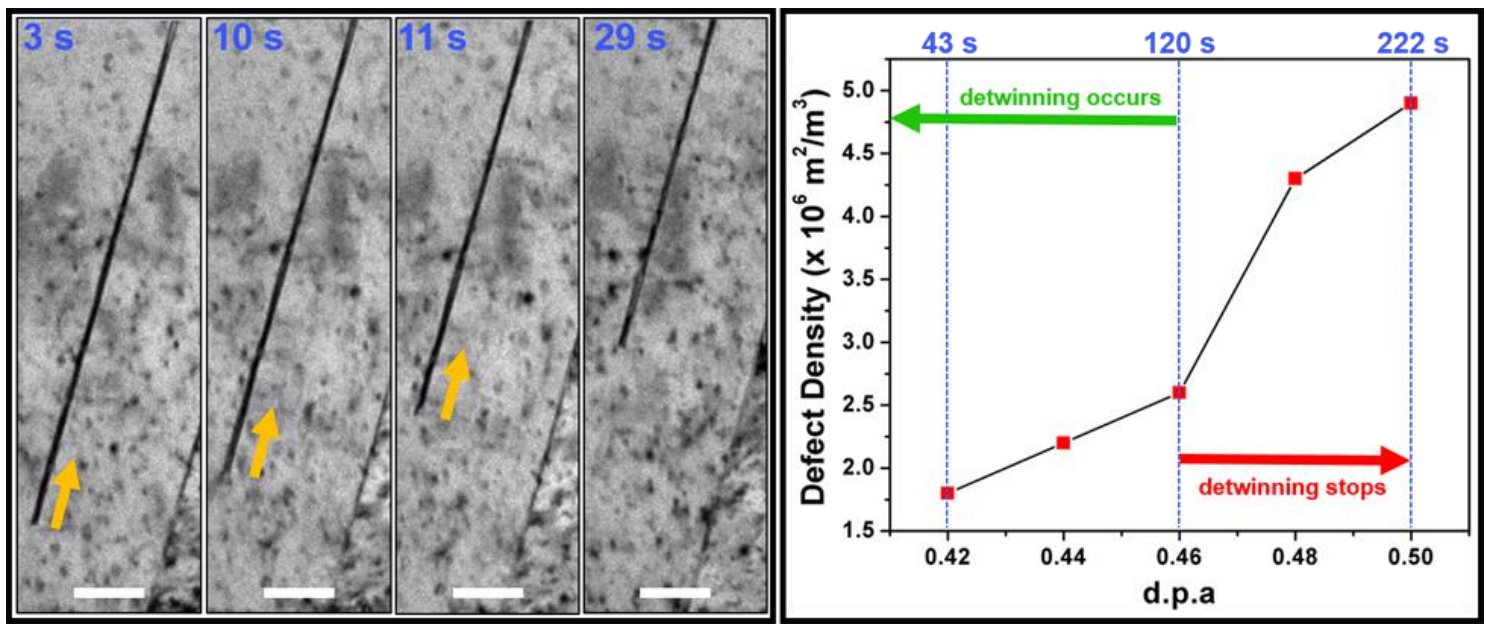

Figure 1. (Left Panel) Bright-field (BF)-TEM snapshots of a nanotwin just before undergoing detwinning via in situ TEM ion irradiation from 0.404 to 0.415 dpa ( 3 s to 29 s). In situ TEM experiment was performed along a two-beam diffraction condition close to [110] zone-axis. Orange arrows depict the direction of detwinning. Scale bar is $50 \mathrm{~nm}$ in all the images. (Right Panel) Plot showing evolution of irradiation-induced defect density in the vicinity of detwinned structure with dose and time. Green and red arrows highlight the regions exhibiting presence and absence of detwinning, respectively.

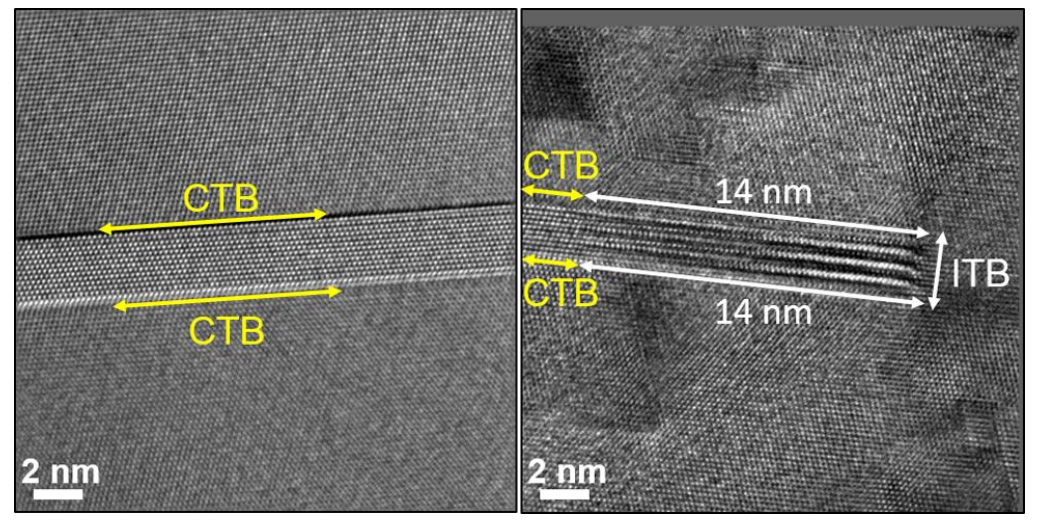

Figure 2. (Left Panel) Atomic-resolution BF-TEM image of a nanotwin before in situ TEM ion irradiation. CTBs are marked in yellow. (Right Panel) Atomic-resolution BF-TEM image of the detwinned structure after in situ TEM ion irradiation dose of $0.5 \mathrm{dpa}$. CTBs and ITBs are marked in yellow and white, respectively. Width of the ITB region is also shown. 